\title{
Sensing Viruses by Mechanical Tension of DNA in Responsive Hydrogels
}

\author{
Jaeoh Shin, ${ }^{1}$ Andrey G. Cherstvy, ${ }^{1}$ and Ralf Metzler ${ }^{1,2, *}$ \\ ${ }^{1}$ Institute for Physics and Astronomy, University of Potsdam, D-14476 Potsdam-Golm, Germany \\ ${ }^{2}$ Department of Physics, Tampere University of Technology, FI-33101 Tampere, Finland \\ (Received 21 October 2013; revised manuscript received 25 February 2014; published 3 April 2014) \\ The rapid worldwide spread of severe viral infections, often involving novel mutations of viruses, poses \\ major challenges to our health-care systems. This means that tools that can efficiently and specifically \\ diagnose viruses are much needed. To be relevant for broad applications in local health-care centers, such \\ tools should be relatively cheap and easy to use. In this paper, we discuss the biophysical potential for the \\ macroscopic detection of viruses based on the induction of a mechanical stress in a bundle of prestretched \\ DNA molecules upon binding of viruses to the DNA. We show that the affinity of the DNA to the charged \\ virus surface induces a local melting of the double helix into two single-stranded DNA. This process effects \\ a mechanical stress along the DNA chains leading to an overall contraction of the DNA. Our results suggest \\ that when such DNA bundles are incorporated in a supporting matrix such as a responsive hydrogel, the \\ presence of viruses may indeed lead to a significant, macroscopic mechanical deformation of the matrix. We \\ discuss the biophysical basis for this effect and characterize the physical properties of the associated DNA \\ melting transition. In particular, we reveal several scaling relations between the relevant physical parameters \\ of the system. We promote this DNA-based assay as a possible tool for efficient and specific virus screening.
}

DOI: 10.1103/PhysRevX.4.021002

\section{INTRODUCTION}

Modern means of transportation, civil aviation traffic in particular, effect extremely rapid global spreading of diseases [1,2], contrasting the much slower spreading dynamics by traveling fronts, for instance, during the Black Death in Europe [3]. Concurrently, new infectious diseases keep emerging constantly, driven by human or ecologic reasons [4], while disease-causing microorganisms are developing various forms of multiple drug resistance [5]. This development puts considerable strain on modern health-care systems, requiring rapid, reliable, and specific diagnosis of infectious agents.

There exist a number of modern techniques to detect viral and bacterial pathogens. However, most of these methods require considerable time and resources. Thus, for the detection of bacteria, typical techniques include the polymerase chain reaction and bacterial culture tests [6]. For viruses, on which we focus in this work, the detection methods include electrochemical [7], optical [8], surface plasmon resonance [9], and biosensor devices [10,11]. Some of them reveal a high single-virus sensitivity and high selectivity to the virus type.

*rmetzler@uni-potsdam.de

Published by the American Physical Society under the terms of the Creative Commons Attribution 3.0 License. Further distribution of this work must maintain attribution to the author(s) and the published article's title, journal citation, and DOI.
Subject Areas: Biological Physics, Interdisciplinary Physics, Soft Matter

Several successful examples of viral detection by bioanalytical chemistry setups have indeed been reported. One of them is the macroscopic swelling of a polymeric acrylamide hydrogel that is cross-linked by specially designed and folded single-stranded DNA (ssDNA) molecules, upon specific binding of influenza viruses $[12,13]$. As demonstrated in that experiment, upon binding of a specific H5N1 influenza strain to the aptamer-containing hydrogel, which coats the surface of a quartz-crystal microbalance biosensor, the linkages of DNA aptamers incorporated in the hydrogel become disrupted and the entire gel structure swells measurably. This highly specific and sensitive detection method for aptamer-based sensors has a number of advantages, as compared to the viral antibody-coated sensors, which are widely implemented for rapid detection of viruses (see, for instance, the assessment in Ref. [14]). Biosensor setups implementing surface plasmon resonance platforms based on antibody-antigen interactions were also shown to be efficient for a selective detection of various influenza, hepatitis B, and HIV viruses [15]. Surface plasmon resonance detection of avian influenza strains with selective binding of DNA aptamers immobilized directly on the sensor surface was developed in Ref. [16], combined with a dot blot assay for a visual detection of viruses in tracheal swab samples.

Applications for use in local health-care centers or even as mobile diagnostic tools require the miniaturization of the 
detection device and should provide a real-time signal without a prior amplification step, be easy to use, guarantee reproducible signals, and be relatively inexpensive. Physical, instead of biochemical and biological, techniques may indeed lead the way towards novel diagnosis methods. One promising example is a relatively inexpensive and compact atomic-force-microscope setup to quickly and relatively cheaply test for bacteria and their response to drugs, which was recently proposed in Ref. [17].

Could we come up with a method to detect viruses via a physical signal, for instance, a mechanical contraction of a matrix such as a responsive polyelectrolyte hydrogel, simply based on molecular interaction of the matrix with the surface of the virus shell? In what follows, from extensive computer simulations and statistical mechanical calculations, we demonstrate that viruses may cause a partial melting of double-stranded DNA (dsDNA) molecules due to the binding affinity of the viral shell for ssDNA (see the illustration in Fig. 1). This partial melting of the double helix leads to an overall contractile, entropic force between the two extremities of the linear DNA chain of the order of several $\mathrm{pN}$. Our results demonstrate that the reaction of viruses with DNA chains embedded in a hydrogel indeed leads to a significant, potentially detectable contraction of the hydrogel.

In our computational analysis, we show that there exists a sharp phase transition of the underlying DNA melting, as a function of the binding affinity of ssDNA to the surface of

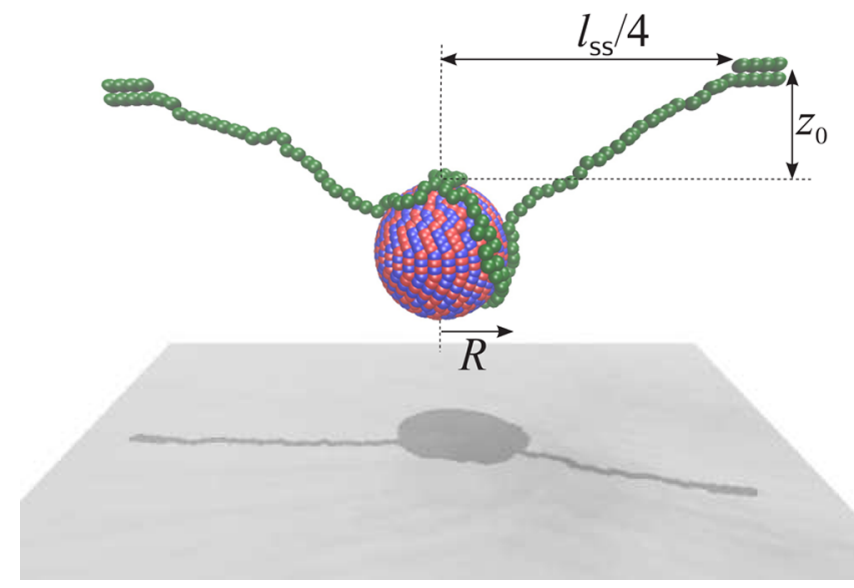

FIG. 1. Schematic of ssDNA-virus binding with the definition of model parameters. Red and blue monomers on the shell correspond to attractive and neutral patches on the "raspberry" surface of the viral capsid. We show one of two ssDNA fragments of a partially denatured, linear DNA (green); at its extremities, we indicate intact dsDNA. A real chain configuration as obtained in simulations for $l_{\mathrm{ss}}=100 \sigma, p=1 / 2, R=6 \sigma$, and $\epsilon_{A}=4 k_{B} T$ is shown. This corresponds to a strong adsorption limit with a progressive wrapping of the flexible ssDNA chain around the attractive sphere. The shell is composed of a spiral of capsid beads running from the pole of the sphere along its surface, with red-blue monomers positioned according to the fraction $p$. See text. the virus. We establish a phase diagram for the denaturation and reveal a scaling relation for the fraction of intact base pairs as a function of the ssDNA-virus binding affinity. Finally, we obtain the contractile force per virus-DNA pair as a function of the binding affinity. Upscaling of these results leads us to our central finding of the effective contraction of a hydrogel equipped with a bundle of DNA chains arranged in parallel in the presence of viruses.

\section{MODELING VIRUS-INDUCED DNA MELTING AND TENSION FORMATION}

In the established thermodynamic models of DNA melting, an alternating sequence of double-helical and denatured segments of different lengths form a partially molten DNA [18-22]. Various levels of DNA coarse graining [23] were implemented to treat the phenomena of DNA melting and DNA strand separation, especially for nanotechnological applications [24]. Once a denaturation bubble is nucleated after overcoming a free-energy barrier $F_{S}$ associated with the cooperativity parameter $\Omega=e^{-F_{s} /\left(k_{B} T\right)} \approx 10^{-5}$, base pairs unzip (and zip closed again) sequentially. DsDNA can be stabilized by some DNA binding cations (protamine, alkaline earth cations) and large ligands [25], cationic lipids and surfactants, crowding agents such as Ficoll-70 [26], basic polypeptides, and DNA-binding proteins (histones), as well as DNA-DNA attractive interactions [27]. Conversely, dsDNA is destabilized by DNA unwinding proteins (gene 32 protein) [28], external DNA twist and supercoiling $[29,30]$, and stretching [31,32], as well as by single-strand DNA binding proteins and intercalators [33,34].

The generation of an entropic force upon melting of prestretched, linear DNA fibers was demonstrated previously. Thus, for highly oriented dense DNA fibers, prestretched by pico-Newton forces, the thermally or chemically induced melting transition of double-helical DNA was shown to trigger macroscopic changes of the fiber length. This melting-induced fiber contraction can reach up to $70 \%-90 \%$ in such mechanochemical studies, solely due to the entropically favored shrinking of the molten segments [35-37]: The highly flexible ssDNA segments have a much smaller equilibrium end-to-end distance compared to the much stiffer dsDNA fragments [38]. Depending on the exact conditions of the solution (salt concentration, temperature, etc.) and the degree of torsional freedom, DNA entropic forces can indeed reach 10 to $40 \mathrm{pN}$ per single DNA molecule in the fiber.

How do viruses come into play? The electrical charge of viruses was already studied in the 1920's [39]. Today, we know that the external surface of the viral capsid, the protein-based shell of viruses, for a number of virus species features strongly nonuniform distributions of electric charges [40-43]. DNA binding to such shells can thus be based purely on electrostatic interactions. For instance, upon infection by an influenza virus, highly cationic haemagglutinin (HA) glycoprotein domains, which form 
protrusions on the viral surface, anchor to negatively charged sialic acid receptors on the host cell surface [44,45]. A number of modern antiflu drugs impede the viral infection proliferation by preventing these electrostatic contacts from forming.

In such a scenario, the DNA-virus binding affinity, the elastic parameters of DNA, and the energy difference between the molten and double-helical states of DNA are all delicately sensitive to the salt concentration and temperature of the ambient solution. For instance, in the range of 0.01 to $0.2 \mathrm{M}$ of monovalent salt, the DNA melting temperature $T_{m}$ exhibits a logarithmic dependence on the salt molarity of the solution [46], leveling off at very large salinities [47]. The electrostatic repulsion of interwound ssDNA strands becomes more pronounced at lower salt levels, thus effectively reducing $T_{m}$. The effects of electrostatic interactions onto the thermodynamics of the DNA melting transition near a charged interface were analyzed in Refs. [48,49]. Thermally induced denaturation of DNA molecules in the presence of an attractive solid interface has been studied theoretically in Ref. [50].

Without external forces and melting, the electrostatically driven adsorption-desorption transition of unconstrained polyelectrolyte chains such as DNA onto oppositely charged curved surfaces has been examined in detail theoretically $[51,52]$ and by computer simulations [53]. The scaling laws for the critical adsorption transition were obtained experimentally as functions of polyelectrolyte chain-surface adhesion strength, salt conditions, surface curvature, and chain stiffness [54]. To tackle such a transition for patchy curved surfaces [55] and adsorbing polymers, as considered here numerically, remains a challenge.

Apart from these solution-sensitive, direct electrostatic attractions between DNA and the surface of the virus, there exists an alternative method to effect binding between ssDNA and the virus capsid. Namely, functionalized, chemically engineered viral shells can bind both dsDNA and ssDNA with different propensities via chemical linkers. For instance, ssDNA can bind better to viral shells covered with ssDNA-binding proteins, as used by viruses to dock to their host cells $[44,45]$. For instance, the DNA strand separation and melting by DNA interactions with the viral capsid proteins of the HIV virus has been demonstrated in Ref. [56]. Thus, by various chemical and biochemical methods, the binding of ssDNA to virus shells may be effected experimentally (see Ref. [57] for the methods of virus surface functionalization with proteins, DNA, and small molecules). Using biochemical linkers, the ssDNA binding may indeed be rendered specific to certain virus types such as influenza, norovirus, HIV, or herpes, and it can also be used for biomedical applications of viruslike particles [58]. At the same time, the binding affinity may reach relatively high values such that the bonds will not dissociate over fairly long time scales.
The presence of viruses can thus favor the (partial) melting of a DNA chain. How can we combine the DNAvirus system with a hydrogel matrix? Hydrogels [59-61] are extensively used in both industrial and medical applications, for instance, for tissue engineering purposes. These cross-linked polymeric materials feature a highly responsive behavior to various external stimuli such as temperature [62], solvent quality, and $\mathrm{pH}$, as well as for detection of various substances [63-66]. Polymeric responsive gels cross linked by DNA strands were demonstrated to be able to shrink and swell as a response to external stimuli [67]. Volumetric changes of up to 100 times in some hydrogels are reported [60] because of electro-osmotic swelling in low-salt solutions (cation accumulation). Viscoelastic hydrogels such as agarose feature a number of rubbery characteristics [68].

We assume that in the hydrogel, individual DNA chains are supported in a prestressed, almost linear configuration and that they are aligned in parallel, as sketched in Fig. 2. The method of fabrication of oriented DNA hydrogel fibers from about 20-kbp-long DNAs has been described in Ref. [69], and the response of these DNA-containing hydrogels to the changes in salt and a $\mathrm{pH}$ stimulus has been systematically studied in Ref. [70]. Perpendicular to their longitudinal axis, the DNA chains occupy a square or hexagonal lattice. We further assume that after adding viruses to the solution, each square or triangular "elementary cell" contains a virus particle. Thus, on average, each virus is associated with one to two DNA double-helices, or two to three ssDNA strands after partial denaturation of the DNA chain (compare Fig. 1). Given the realistic parameters we use in our quantitative analysis below, this means that each ssDNA strand has sufficient accessible area to bind to the surface of the virus capsids, without significant overlap with competing ssDNA segments. In what follows, we consider the interaction of a single dsDNA strand with a given virus capsid. Because of the approximate independence of ssDNA binding to the virus capsids in all cells, we will then be able to upscale our results for the entire DNA

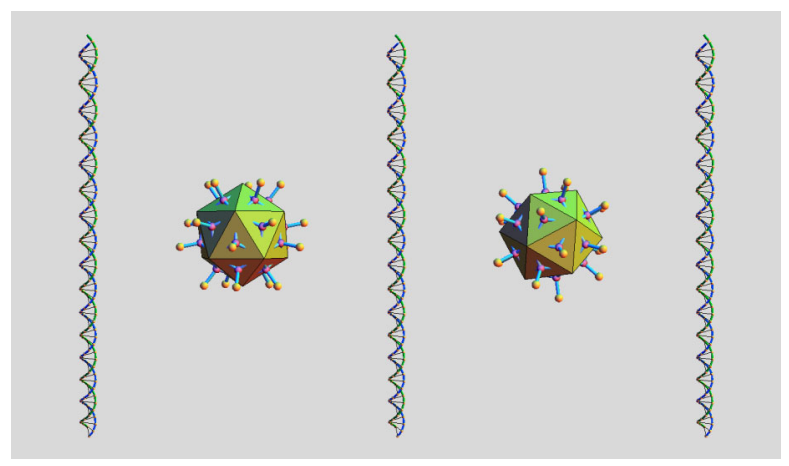

FIG. 2. Schematic of the parallel arrangement of DNA chains with centered virus particles. Thermodynamically, the presence of the virus particles triggers the partial melting of the DNA chains, leading to the configurations shown in Fig. 1. 
bundle-virus assembly in the hydrogel. In this approach, the position of the virus is fixed, reflecting the symmetry of the DNA-capsid configuration. We find that viral particles in the hydrogel-DNA system destabilize dsDNA and effect a macroscopic contraction of the hydrogel matrix.

\section{RESULTS}

\section{A. DNA melting effects contraction of the hydrogel}

As shown in our model configuration in Fig. 1, the surface of the virus is the distance $z_{0}$ away from the axis connecting the two end points of the DNA that are fixed in the hydrogel. The separation between the axis of the DNA molecule from the center of the virus is thus given by $\frac{s}{2}=\left(z_{0}+R\right)$, where, for simplicity, the virus capsid is taken to be spherical with radius $R$, and it is centered with respect to the longitudinal midpoint of the DNA chain between its two extremities incorporated in the hydrogel. In our DNA-virus pair, because of the binding affinity of ssDNA to the capsid, the part of the double helix close to the virus capsid will melt (Fig. 1). The entire DNA molecule is thus composed of two dsDNA segments and two ssDNA segments resulting from the molten middle part.

For now, we concentrate on one of these ssDNA segments, whose length $l_{\mathrm{ss}}=l_{b}+2 l_{f}$, in turn, is made up of the portion $l_{b}$ bound to the viral shell and the two vicinal, unbound ssDNA fragments of length $l_{f}$, each of which connects the bound portion with the two intact dsDNAs. Once such a configuration is established, the resulting entropic strain will be transferred to the end points of the DNA chain. Since the base-pair to base-pair distance is approximately $0.34 \mathrm{~nm}$ in dsDNA, and the phosphatephosphate distance is $0.7 \mathrm{~nm}$ in ssDNA, the maximal ssDNA length available for binding to the virus is approximately 2 times the length of the corresponding dsDNA, provided that the dsDNA fragments can untwist freely upon melting, which may be achieved by the specific anchoring in the hydrogel or by adding a nick close to the extremities of the DNA chain.

For tractability, we neglect effects of DNA torsional rigidity, assuming, in the model, that the azimuthal DNA rotation required for ssDNA binding to a particular virus particle can always be performed. This implies easy DNAtwist rotations that enable DNA bubble formation and propagation of the open DNA state along the molecule as a response to virus binding. Some activity of DNA enzymes nicking one DNA strand can, in principle, achieve this result. It is known that DNA torsion itself triggers DNA base-pair opening. The implications of the external DNA twisting and the degree of supercoiling for circular DNA on the DNA melting characteristics were introduced long ago by Benham [71] (see also the recent single-molecule study [72]).

In our simulations, already-denatured ssDNA chains are equilibrated, and their most probable configurations are analyzed. We distribute attractive monomers on the capsid such that they occupy an area fraction $p$. The interaction free energy $F$ with the virus capsid due to, e.g., electrostatic binding between the DNA chain and the positively charged sites on the capsid, as well as the resulting contraction force $f$ acting on the chain ends, is computed, for different virus radii $R$, DNA separations $z_{0}$ from the virus surface, and ssDNA-virus attraction strengths $\epsilon_{A}$ (the magnitude of the attraction energy per bead). This attraction strength is measured for one monomer of the ssDNA chain that corresponds to a sphere of diameter $\sigma=4 \mathrm{~nm}$ accommodating $\approx 12$ base pairs of the dsDNA or six unpaired bases of the ssDNA. The DNA melting transition necessary for ssDNA formation, triggered by the free-energy gain from binding to the viral surface, is analyzed below as a function of these parameters.

\section{B. Free energy, forces, and adsorption transition}

The relaxation time to reach the equilibrium increases with the length of the simulated ssDNA and for decreasing ssDNA-virus attraction strength. For a ssDNA chain consisting of $n=101$ monomers, the equilibration typically takes approximately $10^{6}$ simulation steps, corresponding to approximately $10 \mathrm{~min}$ on a $3 \mathrm{GHz}$ workstation. For polymer chains of 201 and 401 monomers, the equilibration time takes approximately $3 \times 10^{6}$ and $10^{7}$ simulation steps. Every point in Figs. 3, 5-7 is calculated as an average over at least $10^{4}$ polymer configurations, after equilibration of the system.

It is natural to expect that for large DNA-virus capsid binding strengths, the thermal fluctuations of both the ssDNA and dsDNA chain domains become suppressed. They are effectively pulled out by the binding-mediated force, $f_{A}$, and directed towards the viral particle. For different virus dimensions and DNA-virus separations, this force contains a correction factor $C$ and follows the simple law

$$
f_{A}=C \epsilon_{A} / \sigma
$$

(see Sec. IIID for details). The appendix contains further information on the model potentials used in the simulations, which act between the chain monomers as well as between the polymer chain and the virus surface.

The total free energy of a partially molten DNA double helix is comprised of the sum of entropic and energetic contributions along the equilibrated chain, the interaction energy of the polymer chain with the virus surface $U$, and the DNA melting free energy $F_{\text {melt }}$. The DNA-virus attraction energy scales with the length of bound ssDNA,

$$
U=p B(p) \epsilon_{A} l_{b} / \sigma .
$$

The constant $B(p)$ accounts for multiple contacts that the chain monomers can establish with the attractive sphere 
monomers (valency), where $p$ quantifies the surface fraction of attractive binding spots on the virus (see below). For a fixed ssDNA length $l_{s s}=(n-1) \sigma$, the factor $C$ is given by the slope of the $f_{A}$ versus $\epsilon_{A}$ curve obtained from our simulations. We find, e.g., that for a ssDNA length of $n=151$ monomers with $p=1 / 3$ and $1 / 2$, the values of the constant $C$ are about 2.7 and 3.25 , respectively. The fact that $C$ is greater than unity is because of the multiple contacts of ssDNA beads to attractive monomers of the capsid [the binding valency $B(p)$ ]. For the constant $C$, we come up with the following empirical expression: $C\left(\epsilon_{A}, p\right) \approx$ $p B(p)\left(l_{\mathrm{ss}} / 4\right) /\left[\left(z_{0}+R\right)^{2}+\left(l_{\mathrm{ss}} / 4\right)^{2}\right]^{1 / 2}$.

The DNA melting free energy consists of the free-energy cost for base-pair unstacking, $\Delta F$, and the initiation free energy $F_{s}$ for nucleation of a denaturation bubble,

$$
F_{\text {melt }}=F_{s}+\Delta F l_{\mathrm{ss}} /(6 \sigma) .
$$

For simplicity, all the DNA-related energies are assumed to be independent of the DNA base-pair sequence, its GC content [73], and, most crucially, the length of the ssDNA fragments. In the simulations presented below, we use $\Delta F=0.3-0.5 k_{B} T$ and $F_{s}=10 k_{B} T$. The relatively small $\Delta F$ values for the helix stabilization free energy per base pair mimic some destabilizing conditions of DNA at elevated temperatures (compare to the values of $\Delta F$ per base pair in the standard models of thermal melting, where typically $\left.\Delta F \sim 1 k_{B} T\right)$.

The denaturation equilibrium of dsDNA and the fluctuation spectrum of ssDNA become altered by the ssDNA binding to the viral capsid. The optimal length $l_{\mathrm{ss}}^{\mathrm{opt}}$ of the molten DNA is determined self-consistently from the minimum of the total free-energy functional. As an order parameter for the DNA adsorption transition, we use the fraction of ssDNA bases adsorbed to the viral shell,

$$
\theta=l_{b} / l_{\mathrm{ss}}
$$

The position of the phase-transition boundary crucially depends on the strength of the DNA-virus attraction, the DNA density in the bundle, scaling as $1 /\left[\pi(s / 2)^{2}\right]$, and the virus dimensions $R$. It will be instructive to account for the maximal fraction of ssDNA, $\theta_{\max }$, to be adsorbed because of geometrical constraints of the system (see Figs. 3 and 6). In this state, the flanking ssDNA fragments are fully stretched (strong adsorption limit). The value of $\theta_{\max }=1+2 R / l_{\mathrm{ss}}-\sqrt{1 / 4+\left[2\left(R+z_{0}\right) / l_{\mathrm{ss}}\right]^{2}}$ is a function of the virus size and the DNA-capsid separation.

We implement the (standard) dynamical criterion for adsorption of a ssDNA chain monomer to the virus. When a monomer stays in contact with the surface for more than $50 \%$ of the simulation time, it is considered adsorbed. We assume that a portion $0<p<1$ of the spherical virus surface, composed of monomeric patches as indicated in Fig. 1, is attractive to ssDNA. Figure 1 shows a typical configuration of the virus surface with adsorbed DNA. Generally, attractive monomers may form a single attractive patch or be distributed randomly on the surface, as implemented below. Another possibility is to form a structure of interconnected ridges mimicking a nonuniform distribution of attractive capsid proteins on the virus surface. Note that for partially attractive surfaces, the ssDNA monomers proximal to both attractive and neutral capsid monomers are equally counted adsorbed by the above criterion. In the majority of situations, the ssDNA chain is strongly confined to the capsid surface, leaving the free ends in a fairly stretched conformation. For relatively large adsorption strengths $\epsilon_{A}$ and for chain lengths much longer than the persistence length, $l_{\mathrm{ss}} \gg l_{p} \approx 1 \ldots 4 \mathrm{~nm}$, that we examine in the current study, the effects of a slightly varying ssDNA persistence on the main scaling characteristics of the adsorption-triggered DNA melting transition will not be substantial.

The contractile force $f$ along the DNA axis acting on the dsDNA extremities connected to the hydrogel is due to both entropic ssDNA fluctuations tending to coil up the chain and (partial) adsorption to the virus capsid. To find $f$ for a fixed length $l_{\mathrm{ss}}$ of ssDNA and for different overall DNA end-to-end distance $y_{0}$, we compute the binding energy $U\left(y_{0}\right)$ using the weighted histogram analysis method (WHAM) [74]. This method is a particular implementation of the umbrella sampling method. Then, we evaluate the force as

$$
f=-\frac{d}{d y_{0}}\left[U\left(y_{0}\right)+F_{\text {melt }}\left(y_{0}\right)\right]=k_{y} \delta l .
$$

Here, the elastic constant $k_{y}$ for the displacement $\delta l$ of the DNA ends varies in the range $k_{y} \sim 0.1 \ldots 1 k_{B} T / \mathrm{nm}^{2}$ for different DNA densities and for the Young modulus of a hydrogel, $E \sim 20 \mathrm{kPa}$. This value of $E$ is due to other polymeric components of the hydrogel supporting the incorporated, regular lattice of DNA interacting with viral particles [75].

For a typical DNA density $(s \sim 70, \ldots, 150 \mathrm{~nm})$, we compute the cross-section area per DNA in our cell model as $S \approx \pi\left(z_{0}+R\right)^{2}$. Then, the microscopic contraction of one DNA in the cell via the Young modulus can be related to the relative contraction of the entire material via

$$
\frac{\delta l}{l_{\mathrm{ss}}} \approx \frac{f}{E \pi\left(z_{0}+R\right)^{2}}
$$

This contraction is triggered by ssDNA adsorption to the virus surface and represents the macroscopically measured quantity.

\section{Fixed ssDNA length}

We now present the results of our extensive Molecular Dynamics computer simulations. To that end, we first 
obtain the statistical behavior for the DNA-virus interaction for a fixed length of the available ssDNA fragment, before determining the optimal ssDNA length self-consistently. We note that the relatively high initiation barrier $F_{s}$ for DNA bubble formation prohibits the creation of short molten stretches of ssDNA. Only longer ssDNA fragments are stabilized by binding to the viral capsid.

We find that the fraction $\theta$ of adsorbed segments increases with the adsorption strength $\epsilon_{A}$ once a critical value $\epsilon_{A}^{\star}$ is exceeded. After this transition, the amount of adsorbed ssDNA segments increases up to a saturation plateau given by $\theta_{\max }$ (see Fig. 3). As mentioned above, the geometry of our setup influences the shape of this transition, in particular, the distance $z_{0}$ between the central DNA axis and the surface of the capsid (see Fig. 5). Because of the transition as a function of the adsorption strength, the standard sigmoidal DNA melting curve $\theta(T)$ known for DNA thermal melting in our adsorption-induced melting scenario acquires a kink. According to Fig. 3, the transition of $\theta=\theta\left(\epsilon_{A}\right)$ appears to be of second order. For large viruses and strong ssDNA-virus attraction, nearly all chain monomers are adsorbed, and nonadsorbed ssDNA fragments are therefore fully stretched.

For a fixed DNA-virus separation $z_{0}$, the fraction $\theta$ increases with the capsid radius $R$ as the overall number of available attractive patches on the viral shell increases. In the limit of strong binding affinity $\epsilon_{A}$, the fraction $\theta$ saturates to the geometry-dependent value $\theta_{\max }\left(R, z_{0}\right)$. We observe that smaller viral particles naturally require larger attraction strength for the onset of the denaturation adsorption and yield smaller fractions of bound ssDNA bases, as shown in Fig. 3 and Fig. S1 (Supplemental Material [76]). This effect may potentially be used to differentiate viral capsids of different sizes (selectivity of the detection device).

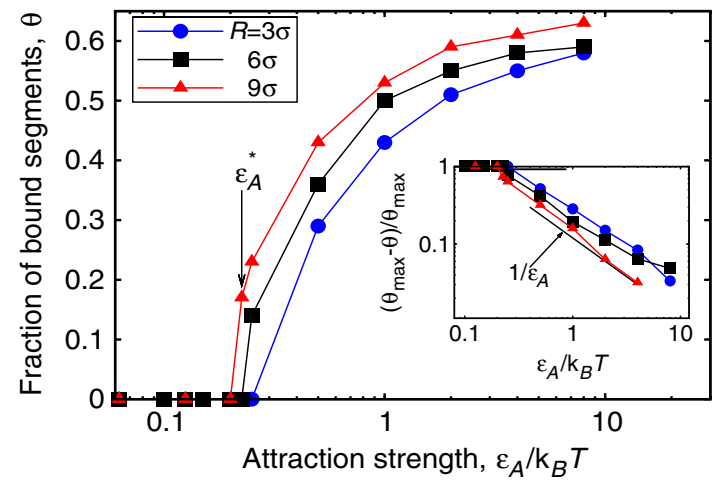

FIG. 3. Fraction $\theta$ of bound DNA base pairs as a function of ssDNA-virus attraction $\epsilon_{A}$ for a uniformly attractive viral particle $(p=1)$ and varying capsid radius $R$. Parameters: DNA-virus distance $z_{0}=8 \sigma$, number of ssDNA monomers $n=101$, ssDNA end-to-end separation $y_{0}=(n-1) \sigma / 2=50 \sigma$. Inset: The residual fraction of nonadsorbed monomers reveals the scaling $1 / \epsilon_{A}$ (solid line). Error bars are comparable to the symbol size.
Interestingly, the decrease of the fraction of nonadsorbed ssDNA bases with the attraction strength fulfills the scaling relation

$$
\frac{\theta_{\max }-\theta}{\theta_{\max }} \sim \frac{k_{B} T}{\varepsilon_{A}}
$$

for sufficiently large values of $\epsilon_{A}$. This universal dependence is illustrated in the inset of Fig. 3. The scaling relation (7) is fully consistent with the extension of a polymer chain in the limit of strong stretching forces. This follows from the relative chain extension $\bar{r} / l_{0}=\operatorname{coth}\left[f \sigma /\left(k_{B} T\right)\right]-1 /$ $\left[f \sigma /\left(k_{B} T\right)\right] \approx 1-1 /\left[f \sigma /\left(k_{B} T\right)\right]$ of a wormlike polymer at large applied forces $f \sigma /\left(k_{B} T\right) \gg 1$. Here, the chain contour length is $l_{0}=(n-1) \sigma$. As the stretching force is due to ssDNA adsorption to the viral capsid, we have $f \sim \epsilon_{A} / \sigma$ and $\left(l_{0}-\bar{r}\right) / l_{0} \sim\left(\theta_{\max }-\theta\right) / \theta_{\max } \sim k_{B} T / \epsilon_{A}$.

In line with our expectations, the distribution of the chain monomers adjacent to the attractive viral capsid surface becomes strongly localized at larger binding affinities (see Fig. 4). For the case $\epsilon_{A}=2 k_{B} T$, the width of this distribution is indeed very narrow. This distribution significantly broadens, and its maximum shifts away from a monomeric distance from the capsid surface when $\left|\epsilon_{A}\right|$ decreases. This is an obvious tradeoff between entropic and enthalpic effects when $\epsilon_{A}$ is comparable to thermal energy.

A natural question to ask is whether, similar to the thermal melting transition, the sharpness of the bindinginduced DNA melting transition increases with DNA length. We study the effect of the ssDNA length in Fig. S2 of Ref. [76], observing that shorter chains require larger adsorption strengths to initiate the DNA-virus binding (at a constant DNA-virus distance). We also observe faster saturation to the geometry-limited value $\theta_{\max }$.

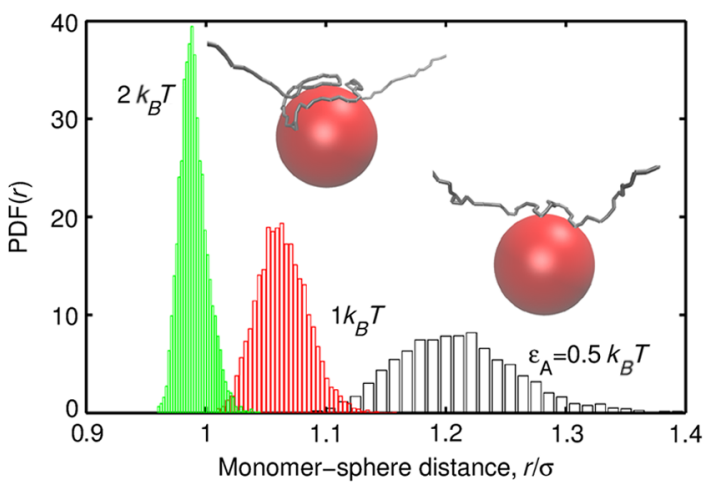

FIG. 4. Probability density function of the radial distance of ssDNA monomers from the capsid surface for different values of the adsorption strength $\epsilon_{A}$, ranging from weak (black) over intermediate (red) to strong binding (green). Parameters are the same as in Fig. 3, and $R=6 \sigma$. In the Supplemental Material [76] we include video files for the ssDNA adsorption process onto the capsid surface for the situations of strong and weak adsorption, for the values $\epsilon_{A}=4 k_{B} T$ and $\epsilon_{A}=0.5 k_{B} T$, respectively. 


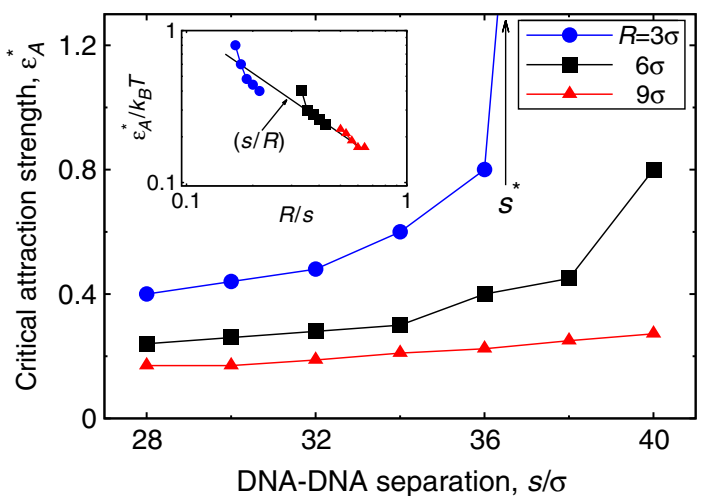

FIG. 5. Critical adsorption strength $\epsilon_{A}^{\star}$ for the onset of ssDNA adsorption, plotted at varying DNA-DNA separations $s$ and virus sizes $R$. The maximal DNA-DNA separation at which the adsorption strength diverges is indicated as $s^{\star}$. Inset: Same set of data sampled at constant $s$ values reveals a scaling $\epsilon_{A}^{\star} \sim s / R$. Parameters: DNA-shell distance $z_{0}=(s-2 R) / 2$; other parameters as in Fig. 3.

However, the transition does not appear sharper for longer ssDNA molecules: Approximately the same number of adsorbed polymer segments are detected at the onset of ssDNA binding at the critical attraction strength $\epsilon_{A}=\epsilon_{A}^{\star}$.

We also find that the critical value $\epsilon_{A}^{\star}$ decreases for larger radii $R$ of the capsid, as shorter DNA-virus separations need to be bridged by the molten DNAs, thus facilitating the adsorption process (see Fig. 5). In comparison, the critical $\epsilon_{A}^{\star}$ for different $R$ with the same $z_{0}$ is nearly identical (not shown). This result indicates small effects of the shell surface curvature on the onset of the adsorption transition. For adsorption strengths well above the adsorption transition, in contrast, the surface curvature effects are vital because they regulate the number of sites available for adsorption for a given chain length $l_{\mathrm{ss}}$ and shell-DNA separation $z_{0}$, as seen from Fig. 3 .

At a critical DNA-DNA separation $s^{\star}=s^{\star}(R)$, the length of the ssDNA segment becomes insufficient to allow adsorption to the capsid. The critical attraction strength at this adsorption-desorption transition of the ssDNA naturally diverges, as shown in Fig. 5. Well above the adsorption transition (e.g., at relatively small $s$ values), the data reveal a distinct scaling of the critical adsorption strength with the capsid radius,

$$
\epsilon_{A}^{\star}(R) \sim s / R=2 z_{0} / R+2 .
$$

This asymptote, shown in the inset of Fig. 5, reveals the geometric competition between the DNA-virus separation $s$ and the radius $R$.

In Fig. 6, we present the results for the fraction $\theta$ of bound ssDNA monomers with a varying fraction $p$ of attractive, randomly distributed capsid monomers. For larger ssDNA-virus attraction strengths $\epsilon_{A}$, smaller $p$ values are sufficient to trigger the DNA adsorption; in

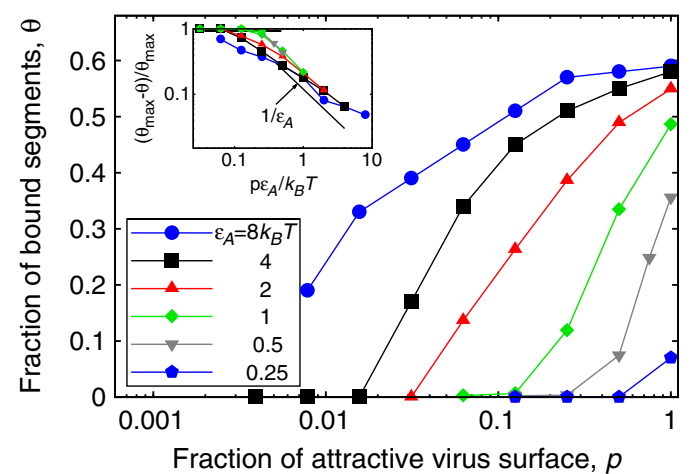

FIG. 6. Fraction of bound ssDNA segments growing with the virus surface coverage by attractive monomers, $p$. Inset: Fraction of nonbound segments exhibiting the universal scaling (7). Other parameters are as in Fig. 3, and $R=6 \sigma$.

other words, the parameter $p$ of the surface coverage by attractive capsid monomers is complementary to the adsorption energy $\epsilon_{A}$ per monomer [77]. The DNA adsorption reveals an apparent second-order continuous transition in the variable $p$. It is thus natural to introduce the generalized parameter $p \epsilon_{A}$ and to reexamine relation (7) obtained above for the uniformly attractive sphere. We observe that the fraction of unbound ssDNA monomers exhibits the analogous scaling behavior (7), substituting $p \epsilon_{A}$ for $\epsilon_{A}$.

\section{Optimal ssDNA length and hydrogel contraction}

In the previous subsection, we studied the adsorption transition at a fixed ssDNA length. Physically, this length is determined self-consistently from the competition of DNAvirus attraction and DNA melting free energy, as detailed in this section.

As the length $l_{\mathrm{ss}}$ of the overall ssDNA segment is varied, we find an optimal length $l_{\mathrm{ss}}^{\mathrm{opt}}$ that corresponds to the minimum of the total free energy. With progressing DNA adsorption, the attractive energy $U$ decreases almost linearly for short ssDNA and appears to saturate for longer chains. This slower increase of $|U|$ is due to a self-repulsion of the segments already adsorbed on the sphere. The melting energy cost grows linearly with the length of molten DNA [see Eq. (3) and Fig. S3 of Ref. [76]].

In Fig. S4 of Ref. [76], we show the optimal ssDNA length for three energy differences $\Delta F$ between ssDNA and dsDNA. The melting transition only takes place if the total free energy of the simulated ssDNA fragment in the molten-adsorbed state is lower than that in the helicaldesorbed state. This defines the minimal bubble size when DNA virus-induced melting becomes energetically beneficial. Below a critical adsorption strength $\epsilon_{A}^{\star \star}$, no DNA melting takes place and $l_{\mathrm{ss}}^{\mathrm{opt}}=0$. For $\epsilon_{A}>\epsilon_{A}^{\star \star}$ the value $l_{\mathrm{ss}}^{\mathrm{opt}}$ starts to grow from a finite value with $\epsilon_{A}$, as shown in Fig. S4. This jumplike behavior is indicative of a first-order transition, in contrast to the continuous change in $\theta$ 
presented in Fig. 6. Smaller $\Delta F$ values favor bubble formation and an earlier onset of DNA opening and thus shift the equilibrium towards the molten DNA state.

The dependence of the optimal ssDNA length on the model parameters obtained from Fig. 6 defines the onset of a longitudinal contraction in the system. Starting from the adsorption strength $\epsilon_{A}^{\star \star}$, the contraction force per DNA increases nearly linearly with the ssDNA-virus adhesion strength (see Fig. 7). The contraction force acting on the ends of the DNA follows the linear scaling with $\epsilon_{A}$ given by Eq. (1). For larger $\Delta F$ values, the equilibrium length of the ssDNA decreases, while its effect on the gel contraction becomes stronger. The effects of thermal fluctuations on the contraction force $f_{A}$ can be recalculated from the uncertainties in the length of melted DNA, systematically exploited in Fig. S4.

Collecting our previous results, we arrive at the main conclusion of this work, the relative contraction $\delta l / l_{\mathrm{ss}}$ of the hydrogel. According to Eq. (6), this relative contraction $\delta l / l_{\text {ss }}$ scales linearly with the contraction force $f$ and is inverse proportional to the Young modulus E. According to Fig. 7, the force $f$ is approximately proportional to the attraction strength $\epsilon_{A}$, thus confirming Eq. (1). For the optimal ssDNA length, the value of $C$ for the fraction of $p=1 / 3$ shown in Fig. 7 is $C \approx 1.9$, which is somewhat smaller than for the situation of shorter and constant ssDNA length treated in the previous section. The physical reason is that for a longer ssDNA length adsorbed on the capsid surface (see Fig. 7 and Fig. S3), the adsorption energy benefit is typically reduced because of progressively stronger self-repulsion of already-adsorbed ssDNA monomers.

The right axis in Fig. 7 shows the corresponding relative contraction $\delta l / l_{\mathrm{ss}}$ effected by a single ssDNA-virus interaction within one of the "elementary cells" of our setup. Accordingly, for the value $E=20 \mathrm{kPa}$ of the hydrogel

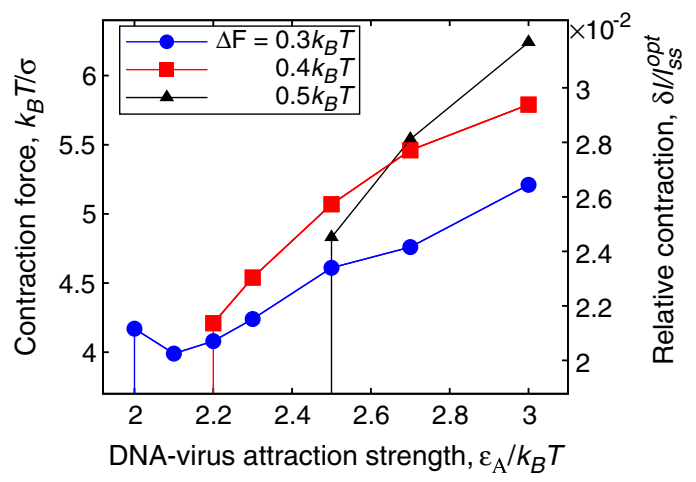

FIG. 7. Contraction force and the relative hydrogel contraction, per one ssDNA bound, as a function of $\epsilon_{A}$. As $\sigma=4 \mathrm{~nm}$, we have that $k_{B} T / \sigma \approx 1 \mathrm{pN}$. Parameters: $R=6 \sigma, z_{0}=8 \sigma, p=1 / 3$, and $F_{s}=10 k_{B} T$. For clarity of presentation, the error bars, as recalculated from Fig. S4 with the help of Eq. (6), are not shown. The overall slope of the $f_{A}$ vs $\epsilon_{A}$ is prescribed by the geometrical factor $C$ in Eq. (1).
Young modulus, this would give rise to a contraction of $3 \%, \ldots, 5 \%$. Assuming that at least two ssDNA per elementary cell contribute [78], the contraction of the entire hydrogel would reach almost $10 \%$. This, however, is a conservative estimate with respect to the relatively high value for the Young modulus $E$ that we chose here. Using softer hydrogels, $E$ can easily be reduced considerably, thus leading to a much stronger response to the viral binding. This macroscopic hydrogel contraction is thus immediately detectable.

\section{DISCUSSION}

Based on extensive computer simulations and statistical analyses, we explored the thermodynamic properties of DNA melting induced by binding of single-stranded DNA to viruses and the effected longitudinal tension buildup in the involved DNA chains. In particular, we determined how a preferable adhesion of ssDNA fragments to the surface of the virus shifts the DNA melting equilibrium and alters the character of the DNA melting transition. We found the critical adsorption strength of ssDNA chains to the attractive viral shell under the conditions of DNA confinement in an elastically responsive polymer matrix of a hydrogel. The statistical properties of the partially molten and partially adsorbed DNA chains obtained from computer simulations are in line with the theoretical expectations. We found several scaling relations for the length fraction of adsorbed DNA, the critical adsorption strength, and, most importantly, the relative shrinkage of the supporting hydrogel.

As a proof of concept, we demonstrated that the presence of viruses in a liquid solution may be detected directly by the mechanical response of a hydrogel, transduced by prestretched DNA chains suspended in the hydrogel. These DNAs partially melt and thus expose ssDNA, which itself binds to the virus shell. This binding can, in principle, be made specific for certain types and sizes of viruses. All ingredients are fairly inexpensive to produce, and such a setup can easily be miniaturized. We believe that this setup is a good candidate for mobile use or for use at local healthcare centers. Alternatively to measuring the mechanical deformation of the hydrogel, one could also envision optical signals due to either DNA melting (similar to molecular beacon setups [79]) or strong local hydrogel deformation. The binding to DNA located in separate domains of the hydrogel can be made specific to certain virus types by biochemically specific functionalized units on the virus.

As a final question, we address a potential method to construct a prestretched DNA bundle in a hydrogel and stabilize the setup. A potential scenario is sketched in Fig. 8. We start with a substrate $\mathrm{S}$ supporting the construction initially. This substrate supports a thin foil layer and vertical pillars $P$. The latter are positioned on the substrate via a pattern etched into the substrate. The pillars $P$ are equipped with binding sites for the DNA. This could, for instance, be achieved with standard biotin-streptavidin 
$\mathbf{F}$
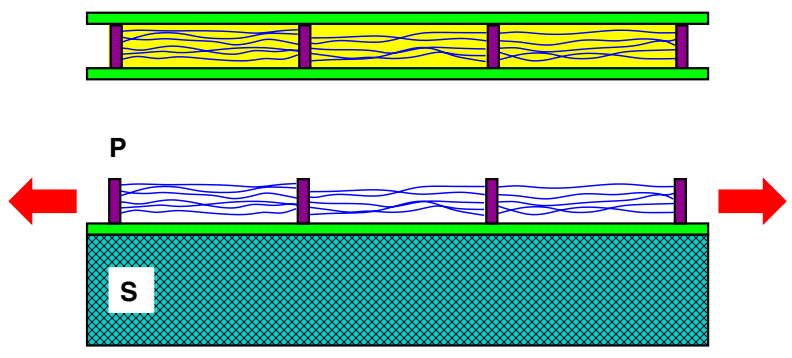

FIG. 8. Sketch of a potential assembly of prestretched DNA bundles in a hydrogel. DNA is attached to pillars $\mathrm{P}$ supported by a substrate $S$, with a thin foil layer $F$ in between. Detaching the DNA bundle-foil construct from the substrate and adding a cover foil as well as the hydrogel (yellow), one arrives at a model system with the properties discussed here. For details, see text.

bonds between the extremities of the DNA construct and the pillar surface. After adding the DNA strands, a second foil $F$ is used as a cover and a hydrogel grown in the volume sandwiched in between the two foils. The construct is then separated from the substrate.

How could the DNA be prestretched in such a scenario? Two different methods would be possible. One is to insert the DNA strands in a somewhat relaxed configuration and then use micromanipulation tools such as optical tweezers to stretch the setup, as indicated by the red arrows. In this method, the pillars are fixated to the foils after applying the stretching force. Another method, which is possibly easier to realize, is to deliver the DNA segments in a nanochannel setup, in which DNA is routinely stretched due to the fact that the channel width is smaller than the persistence length of the DNA [80]. As a result, we would end up with a pseudo-two-dimensional setup. Using extremely thin cover films, the range of contractions predicted in this numerical study should be sufficient to be able to discern surface wrinkles. This potential setup shows that production of DNA-based diagnosis constructs as discussed here could be fairly easy to produce and easy to use as a mobile device.

An elegant alternative approach could be based on advanced DNA origami techniques enabling one to create programmed three-dimensional, self-assembled structures, including hexagonal as well as honeycomb structures of parallel DNA molecules [81] and, in particular, intrinsically prestressed DNA bundles of a well-controlled threedimensional topology [82]. In this latter setup, the interhelical DNA-DNA distances are extremely well controlled by single-stranded DNA fragments of up to several hundreds of bases connecting the arranged double-stranded DNA components. Using these nanofabricated selfassembled DNA structures as a template for a DNAhydrogel biosensor, the diffusion of viral particles into the structure would be possible. Elastic, soft hydrogels which are filling the spaces between the DNA hydrate the DNA lattice and stabilize the canonical B-DNA form. Keeping the system at low temperature before the detection step will keep the DNA lattice in a stable helical state. Exposing this construct to a liquid containing viruses at elevated temperatures will ease the DNA melting transition. Virus particles adhering to ssDNA fragments will compete with the propensity of side-to-side contact formation between dsDNA promoted by the presence of divalent cations added in all DNA origami buffers.

Apart from the direct application to virus sensing, we believe that the DNA denaturation mechanism discussed here and the coupling to a hydrogel matrix will stimulate further research in the fields of soft matter and biological physics. For instance, replacing the stimulus through viruses by coated submicron particles, macroscopic changes of material properties could be affected. The advantage of such a diffusion-controlled mechanism would be the slow and soft response to the stimulus that is often not possible with other methods.

\section{ACKNOWLEDGMENTS}

We thank Professor André Laschewsky for discussions on experimental hydrogel setups. The authors acknowledge funding from the Academy of Finland (FiDiPro scheme to R. M.), the German Research Council (DFG Grant No. CH 707/5-1 to A.G.C.), and the German Ministry for Education and Research (to J. S.).

\section{APPENDIX: SIMULATIONS SCHEME}

We consider the adsorption of ssDNA onto an attractive surface of a viral particle in a simplified model. The ssDNA is modeled by Lennard-Jones (LJ) particles interconnected by finitely extensible nonlinear elastic (FENE) springs of the potential

$$
U_{\mathrm{FENE}}(r)=-\frac{k}{2} r_{\max }^{2} \ln \left(1-\frac{r^{2}}{r_{\max }^{2}}\right),
$$

where $k$ is the FENE spring constant and $r_{\max }$ is the maximum-allowed separation between neighboring monomers. Excluded-volume interactions between the monomers are given by the short-ranged truncated LJ repulsion,

$U_{\mathrm{LJ}}(\epsilon, r)= \begin{cases}4 \epsilon\left[(\sigma / r)^{12}-(\sigma / r)^{6}\right]+\epsilon, & r<2^{1 / 6} \sigma \\ 0, & \text { otherwise. }\end{cases}$

Here, $r$ is the monomer-monomer distance, $\sigma$ is the monomer diameter, and $\epsilon$ is the strength of the potential. We set the parameters to $k=30, r_{\max }=1.5 \sigma$, and $\epsilon=1$. The monomer size is $\sigma=4 \mathrm{~nm}$, close to the Kuhn length of a highly flexible ssDNA. In contrast to dense DNA fibers [37], here we can neglect DNA-DNA interactions because the intermolecular separations exceed the diameter of a typical virus, $2 R \sim 50-100 \mathrm{~nm}$, and the electrostatic forces between individual DNA chains are well screened. 
Performing simulations of a free chain, we checked that the effective ssDNA persistence length in this model agrees well with the known estimate of $l_{p}=1-4 \mathrm{~nm}[83,84]$, and the chain gyration radius follows the Flory law, $\left\langle R_{g}^{2}\right\rangle \sim n^{6 / 5}$, for an excluded-volume or self-avoiding polymer as a function of the number $n$ of monomers. The inset in Fig. S3 indicates that our ssDNA chains obey the scaling for self-avoiding polymers down to the chain length of two beads, showing no indication of persistence. We thus conclude that the effective persistence of these chains is within the range pertinent to ssDNA molecules.

The spherical viral shell of radius $R$ is modeled by a densely packed assembly of LJ beads of size $\sigma$. A finite fraction $0<p<1$ of the monomers, distributed either in a random or an ordered fashion on the viral surface, is attractive for ssDNA segments in their vicinity. The ssDNA-virus attraction is modeled via the same spherically symmetric LJ potential,

$$
U_{\mathrm{PV}}(r)=U_{\mathrm{LJ}}\left(\epsilon_{A}, r\right),
$$

but with a larger cutoff distance $r^{\star}=2.5 \sigma$ to ensure the existence of an attractive potential branch at larger distances and with varying attraction strength $\epsilon_{A}$. The dynamics of the $i$ th chain monomer is described in our molecular dynamics analysis by the Langevin equation

$$
\begin{aligned}
m \frac{d^{2} \mathbf{r}_{i}(t)}{d t^{2}}= & -\xi \mathbf{v}_{i}(t)+\mathbf{F}_{i}^{R}(t)-\sum_{J} \nabla U_{\mathrm{PV}}\left(\left|\mathbf{r}_{i}-\mathbf{r}_{V}-\mathbf{R}_{J}\right|\right) \\
& -\sum_{j, j \neq i} \nabla U_{\mathrm{LJ}}\left(\left|\mathbf{r}_{i}-\mathbf{r}_{j}\right|\right)-\nabla U_{\mathrm{FENE}}\left(\left|\mathbf{r}_{i}-\mathbf{r}_{i \pm 1}\right|\right),
\end{aligned}
$$

where $\mathbf{R}_{J}$ is the radius-vector of the $J$ th capsid monomer from the shell center, $m$ is the monomer mass, $\xi$ the friction coefficient, $\mathbf{v}_{i}$ the monomer velocity, and $\mathbf{F}_{i}^{R}$ represents zero-mean Gaussian noise with component-wise $\delta$ correlation, $\left\langle\mathbf{F}_{i}^{R}(t) \mathbf{F}_{j}^{R}\left(t^{\prime}\right)\right\rangle=6 \xi k_{B} T \delta_{i j} \delta\left(t-t^{\prime}\right)$. The positions of the two polymer ends, $\left(0,0, z_{0}\right)$ and $\left(0, y_{0}, z_{0}\right)$, and of the virus center $\mathbf{r}_{V}=\left(0, y_{0} / 2,-R\right)$ are fixed (see Fig. 1). In the simulations, we set $m=1, \xi=1$, and $k_{B} T=1$. The equation of motion is integrated using the velocity Verlet algorithm [85] with a time step $\Delta t=0.01$.

[1] V. Belik, T. Geisel, and D. Brockmann, Natural Human Mobility Patterns and Spatial Spread of Infectious Diseases, Phys. Rev. X 1, 011001 (2011); L. Hufnagel, D. Brockmann, and T. Geisel, Forecast and Control of Epidemics in a Globalized World, Proc. Natl. Acad. Sci. U.S.A. 101, 15124 (2004).

[2] B. Davoudi, J. C. Miller, R. Meza, L. A. Meyers, D. J. D. Earn, and B. Pourbohloul, Early Real-Time Estimation of the Basic Reproduction Number of Emerging Infectious Diseases, Phys. Rev. X 2, 031005 (2012).
[3] J. V. Noble, Geographic and Temporal Development of Plagues, Nature (London) 250, 726 (1974).

[4] K. E. Jones, N. G. Patel, M. A. Levy, A. Storeygard, D. Balk, J. L. Gittleman, and P. Daszak, Global Trends in Emerging Infectious Diseases, Nature (London) 451, 990 (2008).

[5] A. J. Alanis, Resistance to Antibiotics: Are We in the Post-Antibiotic Era?, Arch. Med. Res. 36, 697 (2005); R. S. Sellar and K. S. Peggs, Management of Multi-DrugResistant Viruses in the Immuno-Compromised Host, British Journal of Haematology 156, 559 (2012).

[6] C. D. Sibley, G. Peirano, and D. L. Church, Molecular Methods for Pathogen and Microbial Community Detection and Characterization: Current and Potential Application in Diagnostic Microbiology, Infect., Genet. Evol. 12, 505 (2012).

[7] F. Patolsky, G. Zheng, O. Hayden, M. Lakadamyali, X. Zhuang, and C. M. Lieber, Electrical Detection of Single Viruses, Proc. Natl. Acad. Sci. U.S.A. 101, 14017 (2004).

[8] B. Brandenburg, L. Y. Lee, M. Lakadamyali, M. J. Rust, X. Zhuang, and J. M. Hogle, Imaging Poliovirus Entry in Live Cells, PLoS Biol. 5, e183 (2007).

[9] S. Wang, X. Shan, U. Patel, X. Huang, J. Lu, J. Li, and N. Tao, Label-Free Imaging, Detection, and Mass Measurement of Single Viruses by Surface Plasmon Resonance, Proc. Natl. Acad. Sci. U.S.A. 107, 16028 (2010).

[10] N. S. Hobson, I. Tothill, and A. P. F. Turner, Microbial Detection, Biosens. Bioelectron. 11, 455 (1996).

[11] M. Schoening and A. Poghossian, Recent Advances in Biologically Sensitive Field-Effect Transistors (BioFETs), Analyst 127, 1137 (2002).

[12] R. Wang and Y. Li, Hydrogel Based QCM Aptasensor for Detection of Avian Infuenza Virus, Biosens. Bioelectron. 42, 148 (2013).

[13] J. Liu, H. Liu, H. Kang, M. Donovan, Z. Zhu, and W. Tan, Aptamer-Incorporated Hydrogels for Visual Detection, Controlled Drug Release, and Targeted Cancer Therapy, Anal. Bioanal. Chem. 402, 187 (2012).

[14] D. Li, J. Wang, R. Wang, Y. Li, D. Abi-Ghanem, L. Berghman, B. Hargis, and H. Lu, A Nanobeads Amplified QCM Immunosensor for the Detection of Avian Influenza Virus H5N1, Biosens. Bioelectron. 26, 4146 (2011).

[15] J. H. Lee, B.-C. Kim, B.-K. Oh, and J.-W. Choi, Highly Sensitive Localized Surface Plasmon Resonance Immunosensor for Label-Free Detection of HIV-1, Nanomedicine 9, 1018 (2013), and references cited therein.

[16] R. Wang, J. Zhao, T. Jiang, Y. M. Kwon, H. Lu, P. Jiao, M. Liao, and Y. Li, Selection and Characterization of DNA Aptamers for Use in Detection of Avian Influenza Virus H5N1, J. Virol. Methods 189, 362 (2013).

[17] G. Longo, L. Alonso-Sarduy, L. M. Rio, A. Bizzini, A. Trampuz, J. Notz, G. Dietler, and S. Kasas, Rapid Detection of Bacterial Resistance to Antibiotics Using AFM Cantilevers as Nanomechanical Sensors, Nat. Nanotechnol. 8, 522 (2013).

[18] D. Poland and H. A. Scheraga, Phase Transitions in One Dimension and the Helix-Coil Transition in Polyamino Acids, J. Chem. Phys. 45, 1456 (1966).

[19] Y. S. Lazurkin, M. D. Frank-Kamenetskii, and E. N. Trifonov, Perspectives Report: Melting of DNA: Its Study 
and Application as a Research Method, Biopolymers 9, 1253 (1970).

[20] J. SantaLucia Jr., A Unified View of Polymer, Dumbbell, and Oligonucleotide DNA Nearest-Neighbor Thermodynamics, Proc. Natl. Acad. Sci. U.S.A. 95, 1460 (1998).

[21] Y. Kafri, D. Mukamel, and L. Peliti, Why is the DNA Denaturation Transition First Order?, Phys. Rev. Lett. 85, 4988 (2000).

[22] D. Jost and R. Everaers, A Unified Poland-Scheraga Model of Oligo- and Polynucleotide DNA Melting: Salt Effects and Predictive Power, Biophys. J. 96, 1056 (2009).

[23] W. G. Noid, Perspective: Coarse-Grained Models for Biomolecular Systems, J. Chem. Phys. 139, 090901 (2013).

[24] P. Sulc, F. Romano, T. E. Ouldridge, L. Rovigatti, J. P. K. Doye, and A. A. Louis, Sequence-Dependent Thermodynamics of a Coarse-Grained DNA Model, J. Chem. Phys. 137, 135101 (2012).

[25] J. M. McGhee, Theoretical Calculations of the Helix-Coil Transition of DNA in the Presence of Large, Cooperatively Binding Ligands, Biopolymers 15, 1345 (1976).

[26] Y. Liu, Y. Shang, H. Liu, Y. Hu, and J. Jiang, Crowding Effect on DNA Melting: A Molecular Thermodynamic Model with Explicit Solvent, Phys. Chem. Chem. Phys. 14, 15400 (2012).

[27] A. G. Cherstvy and A. A. Kornyshev, DNA Melting in Aggregates: Impeded or Facilitated?, J. Phys. Chem. B 109, 13024 (2005).

[28] D. E. Jensen, R. C. Kelly, and P. H. von Hippel, DNA "Melting" Proteins. II. Effects of Bacteriophage T4 Gene 32-Protein Binding on the Conformation and Stability of Nucleic Acid Structures, J. Biol. Chem. 251, 7215 (1976).

[29] J. H. Jeon, J. Adamcik, G. Dietler, and R. Metzler, Supercoiling Induces Denaturation Bubbles in Circular DNA, Phys. Rev. Lett. 105, 208101 (2010).

[30] A. Kabakcioglu, E. Orlandini, and D. Mukamel, Supercoil Formation in DNA Denaturation, Phys. Rev. E 80, 010903 (R) (2009).

[31] M. Rief, H. Clausen-Schaumann, and H. E. Gaub, Sequence-Dependent Mechanics of Single DNA Molecules, Nat. Struct. Mol. Biol. 6, 346 (1999).

[32] A. Hanke, M. G. Ochoa, and R. Metzler, Denaturation Transition of Stretched DNA, Phys. Rev. Lett. 100, 018106 (2008).

[33] K. R. Chaurasiya, T. Paramanthan, M. J. McCauley, and M.S. Williams, Biophysical Characterization of DNA Binding from Single Molecule Force Measurements, Phys. Life Rev. 7, 299 (2010).

[34] P. H. von Hippel, N. P. Johnson, and A. H. Marcus, Fifty Years of DNA “Breathing”: Reflections on Old and New Approaches, Biopolymers 99, 923 (2013).

[35] A. Rupprecht, J. Piškur, J. Schultz, L. Nordenskiöld, Z. Song, and G. Lahajnar, Mechanochemical Study of Conformational Transitions and Melting of $\mathrm{Li}$-, $\mathrm{Na}$-, $\mathrm{K}$-, and Cs-DNA Fibers in Ethanol-Water Solutions, Biopolymers 34, 897 (1994).

[36] J. Piskur and A. Rupprecht, Aggregated DNA in Ethanol Solution, FEBS Lett. 375, 174 (1995).

[37] J. Schultz, A. Rupprecht, Z. Song, J. Piskur, L. Nordenskiöld, and G. Lahajnar, A Mechanochemical Study of MgDNA Fibers in Ethanol-Water Solutions, Biophys. J. 66, 810 (1994).

[38] A. R. Khokhlov and A. Y. Grosberg, Statistical Physics of Macromolecules (American Institute of Physics, New York, 1994).

[39] S. P. Bedson and J. O. W. Bland, A Simple Method for Determining the Electrical Charge Carried by Virus Particles, Brit. J. Exp. Pathol. 10, 67 (1929).

[40] A. Siber, A. L. Bozic, and R. Podgornik, Energies and Pressures in Viruses: Contribution of Nonspecific Electrostatic Interactions, Phys. Chem. Chem. Phys. 14, 3746 (2012).

[41] See http://viperdb.scripps.edu.

[42] A. L. Bozic and R. Podgornik, Symmetry Effects in Electrostatic Interactions between Two Arbitrarily Charged Spherical Shells in the Debye-Hückel Approximation, J. Chem. Phys. 138, 074902 (2013).

[43] A. G. Cherstvy, Electrostatic Interactions in Biological DNA-Related Systems, Phys. Chem. Chem. Phys. 13, 9942 (2011).

[44] N. Arinaminpathy and B. Grenfell, Dynamics of Glycoprotein Charge in the Evolutionary History of Human Influenza, PLoS One 5, e15674 (2010).

[45] Y. Kobayashi and Y. Suzuki, Compensatory Evolution of Net-Charge in Influenza A Virus Hemagglutinin, PLoS One 7, e40422 (2012).

[46] C. Schildkraut and S. Lifson, Dependence of the Melting Temperature of DNA on Salt Concentration, Biopolymers 3, 195 (1965).

[47] R. Owczarzy, Y. You, B. G. Moreira, J. A. Manthey, L. Huang, M. A. Behlke, and J. A. Walder, Effects of Sodium Ions on DNA Duplex Oligomers: Improved Predictions of Melting Temperatures, Biochemistry 43, 3537 (2004).

[48] A. Vainrub and B. M. Pettitt, Accurate Prediction of Binding Thermodynamics for DNA on Surfaces, J. Phys. Chem. B 115, 13300 (2011).

[49] J. Fuchs, J.-B. Fiche, A. Buhot, R. Calemczuk, and T. Livache, Salt Concentration Effects on Equilibrium Melting Curves from DNA Microarrays, Biophys. J. 99, 1886 (2010).

[50] A. E. Allahverdyan, Z. S. Gevorkian, C.-K. Hu, and T. M. Nieuwenhuizen, How Adsorption Influences DNA Denaturation, Phys. Rev. E 79, 031903 (2009).

[51] A. G. Cherstvy and R. G. Winkler, Polyelectrolyte Adsorption onto Oppositely Charged Interfaces: Unified Approach for Plane, Cylinder, and Sphere, Phys. Chem. Chem. Phys. 13, 11686 (2011).

[52] R. G. Winkler and A. G. Cherstvy, Strong and Weak Polyelectrolyte Adsorption onto Oppositely Charged Curved Surfaces, Adv. Polym. Sci. 255, 1 (2013).

[53] Q. Cao and M. Bachmann, Polyelectrolyte Adsorption on an Oppositely Charged Spherical Polyelectrolyte Brush, Soft Matter 9, 5087 (2013); S. C. C. Nunes, T. F. G. G. Cova, and A. A.C.C. Pais, A New Perspective on Correlated Polyelectrolyte Adsorption: Positioning, Conformation, and Patterns, J. Chem. Phys. 139, 054906 (2013); F. Carnal and S. Stoll, Adsorption of Weak Polyelectrolytes on Charged Nanoparticles. Impact of Salt Valency, pH, and Nanoparticle Charge Density. Monte Carlo Simulations, J. Phys. Chem. B 115, 12007 (2011). 
[54] A. B. Kayitmazer, D. Seeman, B. B. Minsky, P. L. Dubin, and Y. Xu, Protein-Polyelectrolyte Interactions, Soft Matter 9, 2553 (2013).

[55] E. Bianchi, R. Blaak, and C. N. Likos, Patchy Colloids: State of the Art and Perspectives, Phys. Chem. Chem. Phys. 13, 6397 (2011).

[56] M. Cruceanu et al., Nucleic Acid Binding and Chaperone Properties of HIV-1 Gag and Nucleocapsid Proteins, Nucleic Acids Res. 34, 593 (2006).

[57] K. G. Patel and J. R. Swartz, Surface Functionalization of Virus-Like Particles by Direct Conjugation Using AzideAlkyne Click Chemistry, Bioconjugate Chemistry 22, 376 (2011).

[58] T. A. P. F. Doll, S. Raman, R. Dey, and P. Burkhard, Nanoscale Assemblies and Their Biomedical Applications, J. R. Soc., Interface 10, 20120740 (2012).

[59] O. Okay, Macroporous Copolymer Networks, Prog. Polym. Sci. 25, 711 (2000).

[60] O. Okay, General Properties of Hydrogels, in Hydrogel Sensors and Actuators, edited by G. Gerlach and K.-F. Arndt, Springer Series on Chemical Sensors and Biosensors Vol. 6 (Springer, Berlin, Heidelberg, 2009), pp. 1-14.

[61] I. Tokarev and S. Minko, Stimuli-Responsive Hydrogel Thin Films, Soft Matter 5, 511 (2009).

[62] C. Bilici and O. Okay, Shape Memory Hydrogels via Micellar Copolymerization of Acrylic Acid and n-Octadecyl Acrylate in Aqueous Media, Macromolecules 46, 3125 (2013).

[63] K. Gawel, D. Barriet, M. Sletmoen, and B. T. Stokke, Responsive Hydrogels for Label-Free Signal Transduction within Biosensors, Sensors 10, 4381 (2010).

[64] M. Quesada-Perez, J. A. Maroto-Centeno, J. Forcada, and R. Hidalgo-Alvarez, Gel Swelling Theories: The Classical Formalism and Recent Approaches, Soft Matter 7, 10536 (2011).

[65] E. Wischerhoff, N. Badi, J. F. Lutz, and A. Laschewsky, Smart Bioactive Surfaces, Soft Matter 6, 705 (2010).

[66] M. A. Cohen-Stuart et al., Emerging Applications of Stimuli-Responsive Polymer Materials, Nat. Mater. 9, 101 (2010).

[67] Y. Murakami and M. Maeda, DNA-Responsive Hydrogels That Can Shrink or Swell, Biomacromolecules 6, 2927 (2005).

[68] M. Ahearne, Y. Yang, A. J. E. Haj, K. Y. Then, and K.-K. Liu, Characterizing the Viscoelastic Properties of Thin Hydrogel-Based Constructs for Tissue Engineering Applications, J. R. Soc. Interface 2, 455 (2005).

[69] C. K. Lee et al., DNA Hydrogel Fiber with SelfEntanglement Prepared by Using an Ionic Liquid, Angew. Chem. 120, 2504 (2008).

[70] S. H. Lee, C. K. Lee, S. R. Shin, S. I. Kim, I. So, and S. J. Kim, The Peculiar Response of DNA Hydrogel Fibers to a Salt and pH Stimulus, Macromol. Rapid Commun. 30, 430 (2009).

[71] C. J. Benham, Torsional Stress and Local Denaturation in Supercoiled DNA, Proc. Natl. Acad. Sci. U.S.A. 76, 3870 (1979).

[72] J. Adamcik, J.-H. Jeon, K. Karczewski, R. Metzler, and G. Dietler, Quantifying Supercoiling-Induced Denaturation Bubbles in DNA, Soft Matter 8, 8651 (2012).
[73] G. Khandelwal and J. Bhyravabhotla, A Phenomenological Model for Predicting Melting Temperatures of DNA Sequences, PLoS One 5, e12433 (2010).

[74] A. Grossfield, "WHAM: the weighted histogram analysis method," version 2.0.7, http://membrane.urmc.rochester .edu/content/wham. The WHAM algorithm to compute the contractile force is equivalent to a brute-force simulation of DNA adsorption at different end-to-end separations, when the molecule is subject to a parabolic potential $W$ that confines its ends, $W=k_{y}(\delta l)^{2} / 2$. In the case of weak DNA-sphere adsorption, however, the WHAM method produces more reliable force estimates, and it is therefore used throughout the paper.

[75] For many hydrogels, the stress-strain relations and the Young modulus are obtained from analysis of elastic deformations and ball indentation [68]. The Young modulus varies in the range $E=0.01, \ldots, 30 \mathrm{kPa}$, depending on the gel crosslinks density, its chemical composition, and temperature [62]. Typically, the modulus grows nearly linearly with concentration of cross-linked polymer chains. Typical values of 5-20 kPa are reported for the aragose gels at $0.5 \%-1.5 \%$ polymer concentrations in the regime in which gel stretching dominates over its bending. The compositions of hydrogels can include various polyelectrolyte chains in different proportions, including a dsDNA.

[76] See Supplemental Material at http://link.aps.org/ supplemental/10.1103/PhysRevX.4.021002 for details on the simulations procedure, additional plots for virus-bound ssDNA length under variation of model parameters, details for computing the gel compression force, and the procedure for determining the optimal length of bound ssDNA.

[77] Some deviations from this scaling for strong adsorption are due to the fact that, in this case, only a small number of polymer monomers are actually in tight contact with the surface. The monomers in polymer loops interconnecting these adsorbed links are, however, also counted as adsorbed, as they are close to the surface. This gives rise to a higher fraction of adsorbed monomers and some deviation from the universal behavior (7) in the strong adsorption limit (see the blue circles in Fig. 6). Smaller $p$ values and larger adsorption strengths are more efficient in binding ssDNAs to the capsid shells.

[78] The average number of ssDNA capable of binding per single viral particle depends on the type of regular model lattices constructed. For instance, for a virus-centered triangular lattice, we get $m=1$; for a square lattice, we get $m=2$; and for a hexagonal virus-centered lattice, $m=4$ ssDNAs are bound per viral particle. These numbers of ssDNA-virus interactions $m$ occur per unit cell of the lattice where the area $S=\pi R_{s}^{2}$ is attributed to each viral particle. The cell radii $R_{S}$ are given for the lattices above as $R_{s}=s \sqrt{\sqrt{3} /(4 \pi)}$, $R_{s}=s \sqrt{1 / \pi}$, and $R_{s}=s \sqrt{3 \sqrt{3} /(2 \pi)}$, respectively. Here, $s$ is the typical DNA-DNA separation in the lattice.

[79] G. Bonnet, O. Krichesvky, and A. Libchaber, Kinetics of Conformational Fluctuations in DNA Hairpin-Loops, Proc. Natl. Acad. Sci. U.S.A. 95, 8602 (1998).

[80] F. Persson, P. Bingen, T. Staudt, J. Engelhardt, J. O. Tegenfeldt, and S. W. Hell, Fluorescence Nanoscopy of Single DNA Molecules by using Stimulated Emission 
Depletion (STED), Angew. Chem., Int. Ed. Engl. 50, 5581 (2011).

[81] S. M. Douglas, H. Dietz, T. Liedl, B. Högberg, F. Graf, and W. M. Shih, Nature (London) 459, 414 (2009); Y. Ke, S. M. Douglas, M. Liu, J. Sharma, A. Cheng, A. Leung, Y. Liu, W. M. Shih, and H. Yan, J. Am. Chem. Soc. 131, 15903 (2009); Y. Ke, N. V. Voigt, K. V. Gothelf, and W. M. Shih, ibid. 134, 1770 (2012).

[82] T. Liedl, B. Högberg, J. Tytell, D. E. Ingber, and W. M. Shih, Self-Assembly of Three-Dimensional Prestressed Tensegrity Structures from DNA, Nat. Nanotechnol. 5, 520 (2010).
[83] H. Chen, S. P. Meisburger, S. A. Pabit, J. L. Sutton, W. W. Webb, and L. Pollack, Ionic Strength-Dependent Persistence Length of Single Stranded RNA and DNA, Proc. Natl. Acad. Sci. U.S.A. 109, 799 (2012).

[84] C. Rechendorff, G. Witz, J. Adamcik, and G. Dietler, Persistence Length and Scaling Properties of SingleStranded DNA Adsorbed on Modified Graphite, J. Chem. Phys. 131, 095103 (2009).

[85] M. P. Allen and D. J. Tildesley, Computer Simulations of Liquids (Clarendon Press, Oxford, England, 1987). 